\title{
La economía política institucional: balance y perspectivas
}

\author{
Fernando GARCÍA-QUERO \\ Universidad de Granada \\ fgquero@ugr.es \\ Fernando LÓPEZ CASTELLANO \\ Universidad de Granada \\ flopezc@ugr.es
}

Received: 05/08/2016

\begin{abstract}
Resumen
Los recientes debates sobre el papel de las instituciones en el desarrollo han mostrado la existencia de dos grandes corrientes teóricas en lo que al pensamiento institucionalista se refiere: la "Nueva Economía Institucional" y la "Economía Política Institucionalista". La primera, encabezada por premios Nobel de Economía como Ronald Coase, Douglass North, Oliver Williamson y Elinor Ostrom, ha sido ampliamente analizada y documentada. En cuanto a la Economía Política Institucionalista, aún puede apreciarse un vacío importante de contribuciones académicas que analicen sus raíces y sus principales aportaciones. El presente artículo trata de superar, en parte, esta laguna mostrando las aportaciones principales de la que, a juicio de algunos autores, puede considerarse como la única corriente "explícitamente institucionalista", la Economía Política Institucional (EPI), que se sitúa en torno a la obra de Ha-Joon Chang. El objetivo del trabajo es doble: ofrecer una aproximación a la EPI y realizar una propuesta de clasificación de las instituciones a partir de las contribuciones de sus principales autores.
\end{abstract}

Palabras clave: neoinstitucionalismo; institucionalismo crítico; instituciones individualizantes; instituciones contextuales; economía política del desarrollo.

\begin{abstract}
The recent debates on the role of institutions in development are revealing two major theoretical currents: the "New Institutional Economics" and the "Institutional Political Economy". The New Institutional Economics, whose most notable representatives are Nobel prizes Ronald Coase, Douglass North, Oliver Williamson and Elinor Ostrom, have been thoroughly examined and analyzed. There is however in the Institutional Political Economy (IPE) an important void of academic works that analyze its roots and its main contributions. This paper tries to fill this gap in that literature. The main goal of this paper is twofold: to offer an approximation of IPE and to suggest a classification and an interpretation of institutions, incorporating the perspectives of the main figures in the field.
\end{abstract}

Keywords: neoinstitucionalism; critical institutionalism; individualizing institutions; contextual institutions; political economy of development.

JEL Classification: B52, Z11, Z18

\section{Introducción}

A partir de los años 90 del pasado siglo se produce un desplazamiento del centro de atención de los teóricos del desarrollo hacia las instituciones. El crecimiento se liga a la existencia de una serie de precondiciones institucionales básicas e, incluso, se propone estudiar el subdesarrollo como un fallo institucional (Bardhan, 2001). Se asume que las políticas públicas y los marcos institucionales son parte fundamental de la ecuación del crecimiento, y que explican, en gran medida, las diferencias de ingresos entre países (North, 1990; Olson, 1996). Este giro desde una "teoría del desarrollo libre de instituciones" a una "nueva teoría del crecimiento" ligada a la Nueva Economía Institucional superó el ámbito académico y alcanzó a las organizaciones multilaterales. En este sentido, el título del informe del Banco Mundial (1998), "El consenso postWashington: las Instituciones Importan", es bien elocuente.

Más allá de esta literatura neo-institucionalista, ligada a la ortodoxia imperante y a la teoría económica convencional, se puede diferenciar otro institucionalismo que parte de una concepción más amplia de las instituciones, la Economía Política Institucionalista (EPI). El objetivo 
del presente trabajo es doble: ofrecer una aproximación a la EPI y proponer una clasificación de las instituciones a partir de las sugerencias de sus principales autores. Con tales fines, se presentan los antecedentes y referentes teóricos de la EPI (sección 2). Seguidamente (sección 3), se exponen las principales aportaciones de la EPI agrupadas en torno a tres epígrafes: las instituciones; el mercado; el Estado, la acción política y el cambio institucional. Posteriormente, en la sección 4, se realiza una propuesta de clasificación de las instituciones a partir de las reflexiones anteriores. Finalmente (sección 5) se exponen las conclusiones del trabajo y se plantean futuras líneas de investigación.

\section{Antecedentes y referentes teóricos}

La publicación en la Cambridge Journal of Economics del artículo "Breaking the Mould - An Institutionalist Political Economy Alternative to the Neo-Liberal Theory of the Market and the State" (2002a) por el economista Ha-Joon Chang puede considerarse como la presentación académica de la Economía Política Institucionalista (EPI). En el artículo se exponían los fundamentos teóricos de un enfoque institucionalista alternativo a la visión convencional en materia de instituciones, agencia humana, rol del Estado y el mercado, cambio institucional y desarrollo económico. Chang continuaba la línea teórica y argumental iniciada casi una década antes tanto por él mismo (Chang, 1994a, 1994b, 2000), como por otros autores (Chang y Rowthorn, 1995; Chang y Evans, 2000; Lazonick, 1991; Evans, 1995; Burlamaqui et al., 2000; Hodgson, 1988, 1993; Block, 1999; Woo-Cumings, 1999). Desde esa fecha hasta la actualidad, se ha ido completando el enfoque con distintas aportaciones (Chang, 2005, 2007, 2011; Di John, 2004; Epstein; 2006; Evans, 2007; Hodgson, 2006, 2007; Lazonick, 2006; Reinert, 2004, 2006). ${ }^{1}$ Los recientes desarrollos de este enfoque institucionalista por Hodgson, Lazonick, Evans, Rutherford, Burlamaqui y Toye, entre otros, pretenden generar un análisis que amplíe la visión convencional de las instituciones como "restricciones" y ofrecer una explicación más sistemática y general del cambio institucional (Chang y Evans, 2005).

Más allá de estos desarrollos, las raíces teóricas de la EPI se remontan al legado intelectual de autores tan variados como Karl Marx (1818-1883), Gustav von Schmöller (1838-1917), Thorstein Veblen (1857-1929), Werner Sombart (1863-1941), Joseph A. Schumpeter (1883-1950), Frank Knight (1885-1972), Karl Polanyi (1886-1694), Herbert Simon (1916-2001), Andrew Shonfield (1917-1981) y John Kenneth Galbraith (1908-2006). ${ }^{2}$ La EPI, al contrario que las denominadas corrientes neoinstitucionalistas ${ }^{3}$, está muy cercana a la "Economía Política Clásica", la "Escuela Histórica Alemana" y la "Vieja Economía Institucional". También guarda cierta afinidad con la biología, la psicología del instinto, la filosofía pragmática (Hodgson, 1998, 2001) la economía evolucionista o la schumpeteriana, huyendo de modelos simplistas de comportamiento racional del individuo y adoptando un enfoque claramente interdisciplinar.

Del viejo institucionalismo, ampliado con los trabajos de John K. Galbraith y Gunnar Myrdal, y los recientes estudios de Greoffrey Hodgson y William Kapp, extrae su énfasis en estudiar la estructura y funcionamiento de los sistemas y procesos económicos, su vocación interdisciplinar y el uso de material empírico histórico y comparativo (Hodgson, 1998, 2003). Tal visión contrasta con la idea de escasez que sustenta la concepción de Robbins, y se aleja mucho de "la mecánica del interés y la utilidad" que defendía Jevons (Kapp, 1968). Como es sabido, Veblen criticó la idea de equilibrio y de individuo utilitarista de la economía neoclásica

\footnotetext{
${ }^{1}$ El libro editado por Chang Institutional Change and Economic Development recopila trabajos teóricos y empíricos de éstos y otros autores, convirtiéndose en una claro ejemplo de esta línea de investigación (Chang, 2007b). Para un análisis de la obra, ver García-Quero y López Castellano (2011).

${ }^{2}$ Pese a que en muchos trabajos de la EPI estos autores no son citados explícitamente, una lectura más exhaustiva permite detectar la influencia de sus orientaciones teóricas y metodológicas. En el trabajo de Chang (2002) se aprecia este hecho puesto que, sin citar a Marx o a Veblen, se intuyen muchas de sus principales ideas, en especial en lo que se refiere a la interconexión entre instituciones y motivaciones (ver Chang, 2002, p.11).

${ }^{3}$ En los trabajos de López Castellano y García-Quero (2012) y (2013) se comparan en tono crítico ambas corrientes, la Nueva Economía institucional (NEI) y la Economía Política Institucional (EPI).
} 
y marginalista y, ante el individualismo metodológico -de los marginalistas- y el holismo metodológico -de los historicistas y de las versiones más esquemáticas de Marx- propuso el dualismo metodológico, que reconoce una relación causal recíproca entre los individuos y el entorno institucional (Hodgson 1998; Galbraith 1987; Mayhew 1998; Rutherford 1994).

De la Escuela histórica extrae su crítica a la abstracción y al método deductivo de la metodología clásica y marginalista, su apuesta por el análisis histórico y estadístico; su rechazo del laissez-faire y de la idea del interés individual como regulador de la acción económica. Como aquélla, se plantea el tema de la especificidad de los fenómenos económicos y la necesidad de que la teoría económica sea más sensible a la diversidad de situaciones históricas y geográficas existentes en la vida económica y social. También comparte la idea de Schmoller de un Estado intervencionista en materia social y garante del principio de justicia redistributiva. Por último, de la visión evolutiva schumpeteriana, ampliada posteriormente por Simon, Nelson y Winter y Reinert, adopta el argumento de que la innovación es un elemento fundamental del desarrollo económico (Lazonick, 1991).

Estas influencias hacen que la EPI no sea un cuerpo unificado de pensamiento, metodología y programa de investigación, puesto que toda su fundamentación teórica concibe la economía como un sistema abierto y dinámico que enfatiza el proceso y no el equilibrio. Además, hace hincapié en los hábitos, las instituciones -su origen y evolución- y en las relaciones de poder (Polanyi, 1944). El sistema es interpretado como un todo, donde las instituciones no se pueden disociar del proceso gradual e histórico de cambio económico, del que son al mismo tiempo consecuencias y componentes fundamentales (Veblen, 1899, 1914).

La apuesta por la multidisciplinariedad y el alto grado de apertura teórica no impide que la EPI parta de unos postulados ampliamente compartidos. La EPI rechaza los supuestos metodológicos del análisis neoclásico, tales como la racionalidad instrumental -homo æeconomicus-, el individualismo metodológico, la cláusula ceteris paribus, la noción de economías externas, el intercambio voluntario o el criterio de bienestar individual. ${ }^{4}$ La EPI niega la existencia de teorías generales y apuesta por la construcción de guías de aproximación a problemas específicos en contextos concretos, a partir de estudios institucionales e históricos basados en la experiencia. La idea es acumular conocimiento y experiencia real, permitiendo crear sinergias, vínculos, interdependencias y simbiosis con otros campos y dentro de los sistemas económicos, que permitan captar diferencias cualitativas entre distintas acciones económicas. La EPI realiza una propuesta normativa basada en la recomendación de políticas económicas sustentadas en la experiencia real.

\section{Las aportaciones de la Economía Política Institucional}

En este apartado se exponen las principales aportaciones de la Economía Política Institucional, agrupadas en torno a tres subepígrafes: las instituciones; el mercado; el Estado, la acción política y el cambio institucional.

\subsection{El rol constitutivo de las instituciones y de las motivaciones}

La EPI parte de una visión más amplia de las instituciones que la que ofrece la corriente neoinstitucionalista, puesto que trasciende su papel como reglas que restringen y condicionan el comportamiento de los agentes. ${ }^{5}$ Para la EPI, las instituciones también forman parte de las motivaciones, haciendo que los individuos las interioricen y se cambien a sí mismos. A su vez, las personas interactúan con el mundo mediante un esquema (unidad organizativa) que está en continua transformación.

\footnotetext{
${ }^{4}$ Como subraya Galbraith $(1955,1967)$, el paradigma del equilibrio perfectamente competitivo es del todo inadecuado para interpretar las economías contemporáneas. Para un análisis riguroso sobre cómo la Vieja Economía Institucional contradice estos supuestos ver Rutherford (1994) y Toboso (1997).

${ }^{5}$ Como conjunto de reglas, las instituciones restringen y condicionan el comportamiento de los agentes; como normas, creencias y valores, motivan a los agentes al seguimiento de las reglas; como organizaciones, permiten la articulación de la acción colectiva y la interiorización de las reglas y motivaciones en las conductas individuales (North, 1990, 2005).
} 
La visión de las instituciones de la EPI implica, por tanto, una nueva interpretación de las motivaciones y de la acción individual. Para esta corriente, las motivaciones que guían la acción individual no son estables, preformadas, egoístas, ni racionales, sino variadas, y responden a un espectro amplio de impulsos de muy diversa índole (Frey 1997) ${ }^{6}$. También niega el supuesto de preferencias egoístas del comportamiento como único punto de partida posible, y sostiene que los individuos pueden estar motivados por el egoísmo más extremo o el más puro altruismo (Lewis y Steinmo, 2011). ${ }^{7}$

A partir de estas premisas, se definen las instituciones como patrones sistemáticos integrados por expectativas compartidas, presupuestos no cuestionados, normas aceptadas, cosmovisiones, códigos morales, valores y rutinas de interacción (Chang y Evans, 2000). Esto significa que las instituciones motivan una regularidad en el comportamiento individual y social e influyen poderosamente en la conformación de las motivaciones de los individuos y actores sociales interrelacionados.

\section{2. El mercado como institución}

La EPI adopta un enfoque de economía política en el tratamiento y análisis del mercado; de ahí que niegue toda posibilidad de estudiarlo al margen de lo político. Todo mercado y toda visión del mismo, en primera y en última instancia, se basan en un conjunto particular de creencias políticas que no pueden reclamar superioridad teórica sobre otro conjunto de creencias. La racionalidad del mercado sólo se entiende si es definida con referencia a una determinada estructura institucional, lo que la convierte en producto político (Vira, 1997). La función de la EPI en este campo es visibilizar e investigar todas estas relaciones e implicaciones. A tal fin, propone detectar y analizar: la estructura de derechos y obligaciones subyacentes para todos los participantes que intervienen en la transacción; la estructura de derechos y obligaciones subyacentes para los que sin intervenir pueden recibir efectos positivos o negativos de la misma (externalidades); la estructura de poder y las instituciones implicadas, lo que vale decir los agentes (individuos u organizaciones) que tienen capacidad de cambiar el sistema de incentivos, y los modelos mentales creados a partir de contexto ideológicos o culturales concretos.

Para la EPI, en fin, el mercado no es el eje central del intercambio sino una institución más de un sistema económico configurado por otras muchas que interaccionan y se modifican entre sí (Polanyi, 1944). Los mercados libres, eficientes, ideales y perfectamente competitivos, que llevan al equilibrio eficiente en términos sociales, no existen. Los mercados no están dados sino que se crean por acciones individuales en un principio y en un determinado momento por acciones políticas, definidas todas ellas por un abanico amplio de instituciones que encierran derechos, obligaciones e ideologías diferentes. En definitiva, a partir de la idea que el mercado es una construcción social y política se definen unos objetivos, se crean unas instituciones en función de esos objetivos y se evalúa su grado de consecución (Chang, 2002). Según esto, lo que para unos economistas es un fracaso, para otros puede ser un éxito organizativo (Lazonick, 1991). El éxito o el fracaso de las instituciones tienen que evaluarse, por tanto, de acuerdo al grado de cumplimiento de los objetivos perseguidos y no a la forma que tengan dichas instituciones.

\subsection{El Estado, la política y el cambio institucional}

La interpretación de la EPI del papel que adopta el Estado y la política en el desempeño de los países es muy diferente de las visiones convencionales, representadas por la Economía del bienestar y la Economía neoclásica. La Economía del bienestar justifica la intervención del Estado en la actividad económica para subsanar los fallos de mercado que la alejan de un óptimo eficiente socialmente. Por su parte, la Economía neoclásica critica la concepción utilitarista de la Economía del Bienestar, al entender que sacrifica la libertad individual en aras de la eficiencia

\footnotetext{
${ }^{6}$ Este punto de partida ha sido demostrado por muchos autores, algunos de ellos no relacionados con la EPI y vinculados a enfoques ortodoxos (Simon, 1983; Mantzavinos et al., 2001; North, 2005).

${ }^{7}$ En relación a este tema, Veblen señalaba que el espíritu del hombre es potencialmente noble.
} 
económica (Buchanan 1986). Desde esta perspectiva, la libertad individual es la que lleva al hombre a crear instituciones eficientes ("racionalidad constructiva", Hayek, 1949). Toda intervención estatal, con independencia de su índole, está condenada al fracaso, debido a los problemas en los que incurre -costes de información, ineficiencia organizativa, incertidumbre, búsqueda de rentas- (Alt y Sheple, 1990). En definitiva, la intervención estatal estaría al servicio de los intereses particulares de los grupos políticos, de ahí que se califique de inmoral, dañina y corrupta, y al mercado de único orden deseable y espontáneo (Burton, 1983).

Desde la posición teórica de la EPI y de su visión de las instituciones, no existe ninguna contrastación empírica que demuestre de forma contundente que la intervención estatal sea ineficiente, corrupta o dañina. Por el contrario, sí existen muchas referencias a intervenciones estatales eficientes, y positivas para la economía y la mejora de las libertades individuales. ${ }^{8} \mathrm{La}$ EPI, rebatiendo el argumento austriaco, demuestra mediante la evidencia histórica que las intervenciones estatales han jugado un papel destacado en la construcción de los mercados (Schmoller, 1872; Reinert, 2007). Para este enfoque, la política es un proceso estructurado institucionalmente (March y Olsen, 1991), y la aparente espontaneidad del mercado no es real, porque su construcción requiere del concurso de los estados.

Según Chang (2002), las instituciones inciden en el proceso político de tres maneras: en la percepción verdadera que los políticos tienen de sus intereses; en la opinión de las personas sobre los temas objetivos, legítimos y deseables para la política; en cómo los individuos perciben la legitimidad de las acciones políticas. Los políticos pueden integrarse en la vida pública comprometiéndose con valores no egoístas para lograr el bienestar social, sin embargo al estar en una esfera "explícitamente pública" pueden verse afectados por incentivos que orienten su comportamiento hacia otros fines. Ellermann (1999) denomina a este hecho "atrofia de la motivación intrínseca”, al describir como los burócratas pueden cambiar motivaciones desinteresadas por otras en contra del bienestar general.

Aunque las instituciones tienden a perpetuar ciertas pautas de interacción humana, la acción política tiene un margen de actuación significativo. Para la EPI el cambio institucional supone un giro en las reglas que constriñen o incentivan los comportamientos sociales pero también implica una transformación de las visiones del mundo que subyacen en las estructuras institucionales (Chang y Evans, 2005). Lewis y Steinmo (2011), en línea con lo apuntado por Hodgson (2002), afirman que actualmente existe un amplio consenso en torno a que las instituciones, las ideas y el ambiente cambian en un proceso coevolutivo.

\section{Una propuesta de clasificación de las instituciones}

En este apartado se va a ensayar una propuesta de clasificación de las instituciones a partir de las reflexiones expuestas a lo largo de los epígrafes anteriores. Se puede afirmar que existen dos tipos de instituciones, en función de si operan dentro del individuo, formando parte de sus modelos mentales, o fuera de él: "instituciones individualizantes" e "instituciones contextuales" (figura 1). Las "instituciones individualizantes" forman parte de los individuos y son inherentes a ellos, pudiendo modificar y afectar a sus motivaciones y comportamientos. ${ }^{9}$ Como se aprecia en la figura 1, estas instituciones tienen dos partes que se complementan e interrelacionan: una de naturaleza restrictiva, que constriñe ("constraints"), y otra de naturaleza integral, que habilita

\footnotetext{
${ }^{8}$ Revaluando las experiencias históricas de casi la totalidad de los considerados de renta alta (EE.UU. Reino Unido, Japón, Alemania, Bélgica, Suecia, etc.) han logrado el estatus de países desarrollados atravesando periodos de fuerte intervención estatal (List, 1885 [1841]; Reinert, 1995; Chang, 2002b). variando en tiempo y espacio el foco, el grado y el modo de dicha intervención ("diversidad institucional del capitalismo"; Chang, 1997, Albert, 1991; Berger y Dore, 1996).

${ }^{9}$ Esta idea está muy cercana a la psicología cognitiva, concretamente a la teoría del conocimiento (Piaget, 1977; Flavell, 1992; Case y Okamoto, 1996), al enfoque del procesamiento de información y al enfoque cognitivo de la neurociencia. Piaget (1977) argumenta que en este proceso el individuo se ajusta a la nueva situación pasando por dos fases: asimilación (aceptar la información nueva e incorporarla a las estructuras cognitivas existentes) y acomodación (cambio en las estructuras cognitivas propias para adecuar el conocimiento nuevo).
} 
("enabling"). ${ }^{10}$ Ambas partes son las que otorgan a las instituciones lo que Chang y Evans (2000) denominan su "rol constitutivo". Este rol hace que los modelos mentales que configuran las motivaciones y preferencias de los individuos varíen y estén determinados por las instituciones.

Figura 1: Clasificación de las instituciones

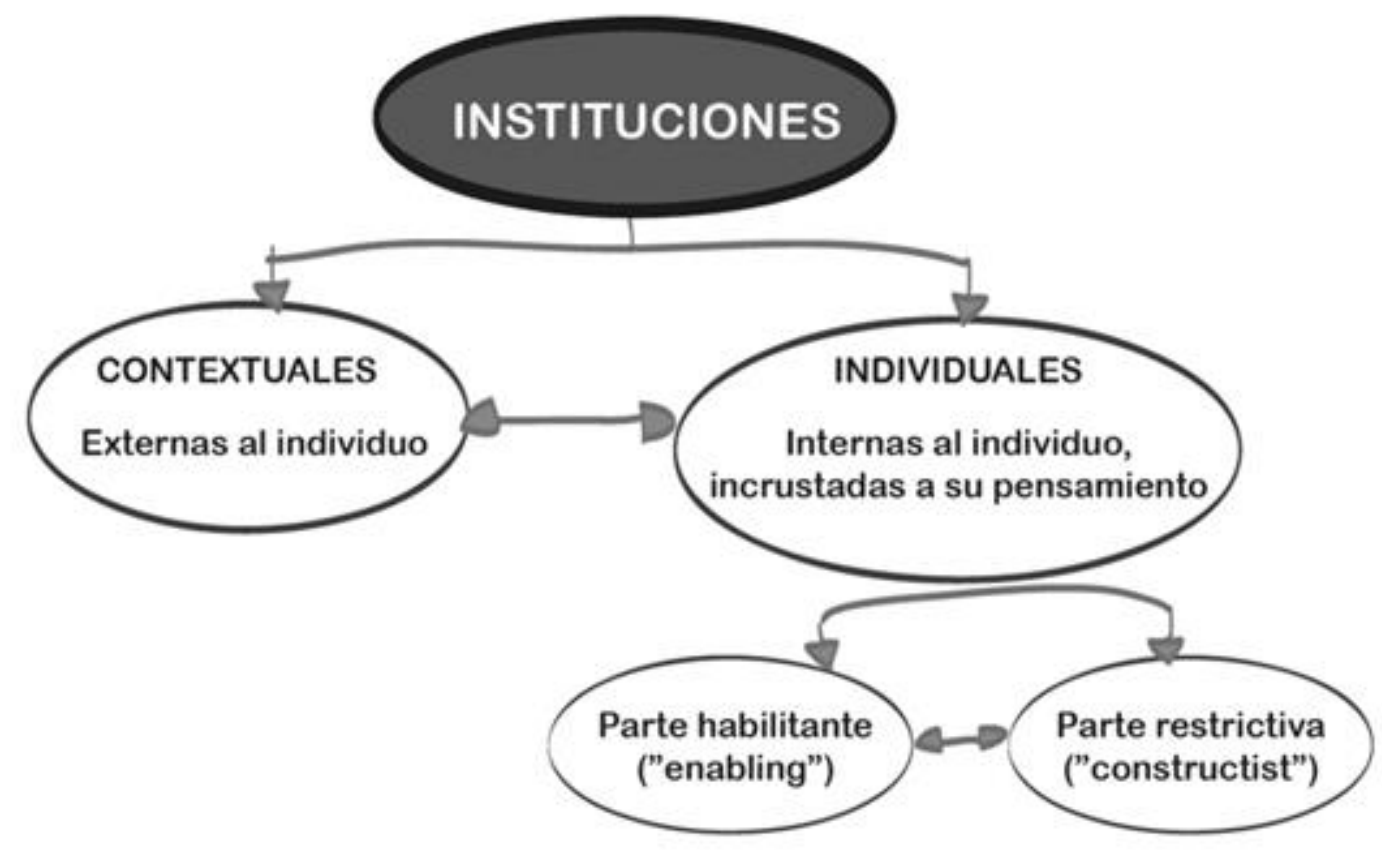

Fuente: García-Quero 2013

De otro lado, los cambios en las motivaciones son cualitativos, dado que una persona en su interacción con el resto, con el entorno y a través de diferentes procesos, va configurando un nuevo modelo mental que sustituye al anterior. Es aquí, donde entra en consideración el segundo tipo de instituciones, las "instituciones contexto", entendiendo por tal el entorno social, familiar, geográfico, comunitario, histórico, cultural, étnico e ideológico, y tecnológico, así como el sistema de incentivos y sanciones, a los que los individuos se enfrentan. ${ }^{11}$ Los estímulos que las instituciones "contexto" provocan en los individuos transforman las instituciones "individualizantes" y producen cambios en sus modelos mentales. El contexto institucional o marco institucional es, por tanto, decisivo en la determinación de los modelos mentales de las personas. ${ }^{12}$ Los seres humanos están formados por instituciones intrínsecas, internas e incrustadas a ellos mismos ("individualizantes") e instituciones extrínsecas y externas a ellos ("contextuales"). Ambas determinan sus modelos mentales y por ende sus comportamientos, conduciendo a que el comportamiento de un individuo o una organización se guíen por el egoísmo, el altruismo, la empatía, el interés por lo público o cualquier otro tipo de motivación.

\footnotetext{
${ }^{10}$ La parte habilitante se correspondería con lo que Hodgson y Veblen denominan hábitos, que configuran el material constitutivo de las instituciones (Hodgson, 2006). En los trabajos de Simons (1983) y Hodgson (1988) aparecen alusiones a estas ideas.

${ }^{11}$ Las instituciones contextuales corresponden con las que North, en la clasificación más extendida de las instituciones, considera como instituciones formales e informales (North, 1990).

${ }^{12}$ Este segundo tipo de instituciones y cómo afectan a los individuos se acerca mucho a los planteamientos conductistas y sociales-cognitivos de la psicología del aprendizaje, así como a las teorías psicológicas contextuales.
} 
Como se aprecia en la figura 2, dos sujetos inmersos en el mismo marco de instituciones "contextuales" han generado dos tipos de preferencias muy diferentes, que se sitúan en los dos polos posibles señalados por Lewis y Steinmo (2011): el egoísmo extremo y el puro altruismo. Las personas formas sus motivaciones y preferencias mediante un proceso endógeno, que Hodgson denomina institucionalización del individuo (Hodgson, 1988) y que no es independiente del contexto social, comunitario e histórico. La acción racional de los individuos no tiene cabida en esta orientación; las instituciones no son producto de la elección racional hecha por individuos interesados en ganancias materiales. Esto hace que dependiendo del contexto pueda ser mejor un tipo u otro de instituciones, negándose así la solución óptima (Hodgson, 2001).

Figura 2. Diferentes personas, diferentes motivaciones

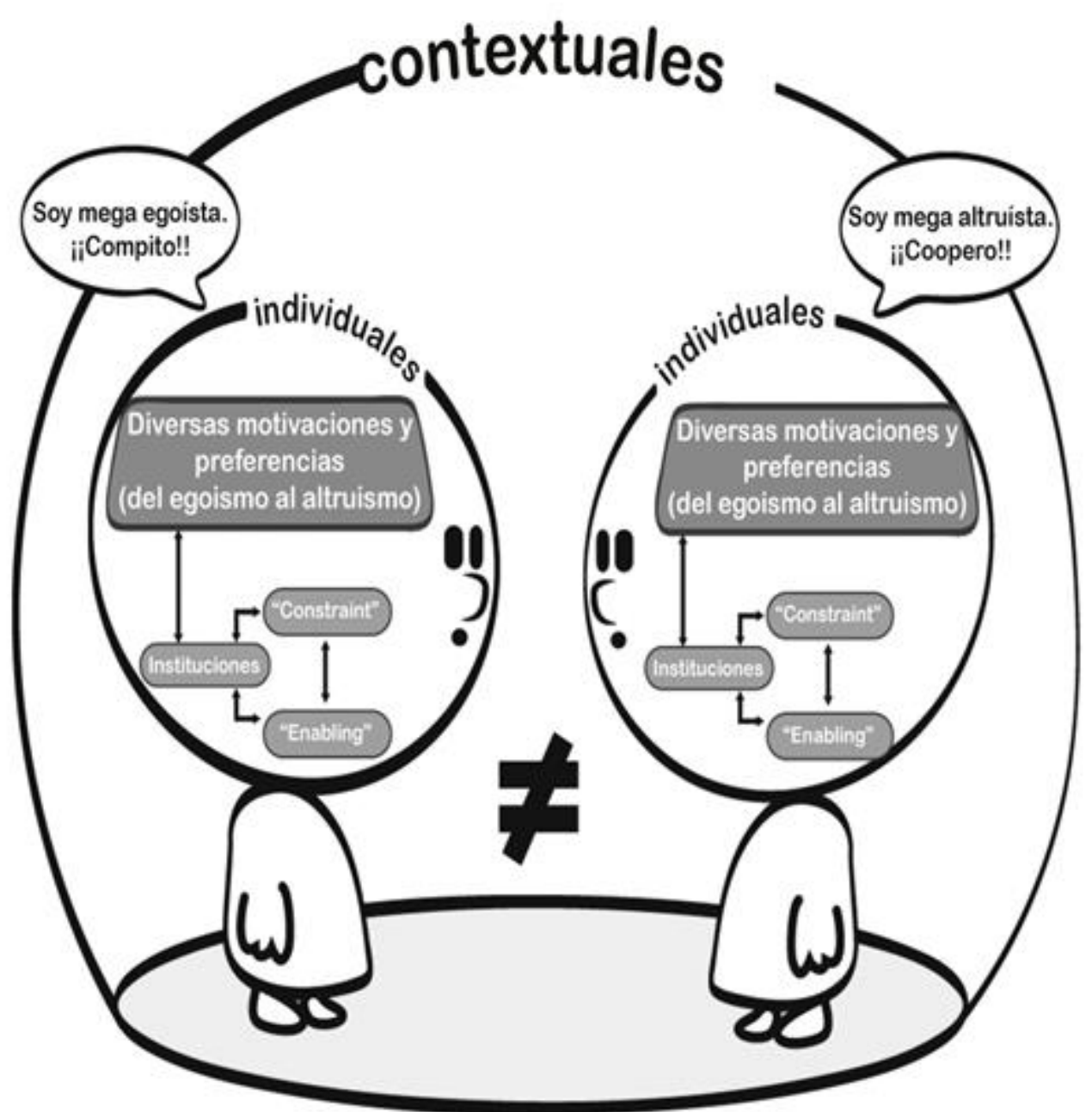

Fuente: García Quero, 2013.

Todas estas reflexiones suscitan una serie de interrogantes sobre la causalidad entre las instituciones individualizantes y las instituciones contextuales. Aunque para la EPI esta cuestión carece de relevancia, la resuelve subrayando que la relación entre motivaciones e instituciones es bidireccional. Herencia, modelos mentales y contexto van de la mano, sus influencias son bidireccionales: las personas cambian su mundo incluso cuando éste las cambia a ellas (Parke et al., 1994). La relación entre una persona y el entorno cotidiano es regular, activa y bidireccional, pudiendo entrar en juego procesos que son afectados por contextos más remotos de los cuales la persona puede incluso no haberse percatado (Bronfenbrenner, 1986; Bronfenbrenner y Morris, 1998). 
Este proceso es lo que ha llamado Hodgson (2000) "causación reconstitutiva descendente". ${ }^{13}$ $\mathrm{Al}$ igual que las instituciones cambian a los individuos, los individuos cambian las instituciones. No hay una relación unidireccional y determinista entre instituciones e individuo. Desde esta visión se demuestra que los patrones de comportamiento se pueden modificar, alterando las motivaciones a través de las instituciones "contextuales" en primer lugar, y de las "individualizantes" en segundo término. Las instituciones individualizantes pueden cambiarse afectando a su parte restrictiva ("constructist") mediante incentivos y sanciones; o a su parte constitutiva ("enabling") mediante exhortación ideológica, educación y valores. ${ }^{14}$ En este sentido, aunque un mismo contexto afecte a todos los individuos no lo hará de igual modo a cada uno de ellos. La experiencia propia que cada persona tenga en su relación con el entorno será única. Es por ello que pueden existir coincidencias entre algunas partes de las instituciones intrínsecas, principalmente ligadas a su ámbito restrictivo, pero nunca habrá dos motivaciones totalmente iguales. Cada individuo crea instituciones de acuerdo a su experiencia propia de interacción. En la siguiente figura (3) se representa esta idea.

Figura 3. Diferentes personas, diferentes procesamientos, diferentes comportamientos

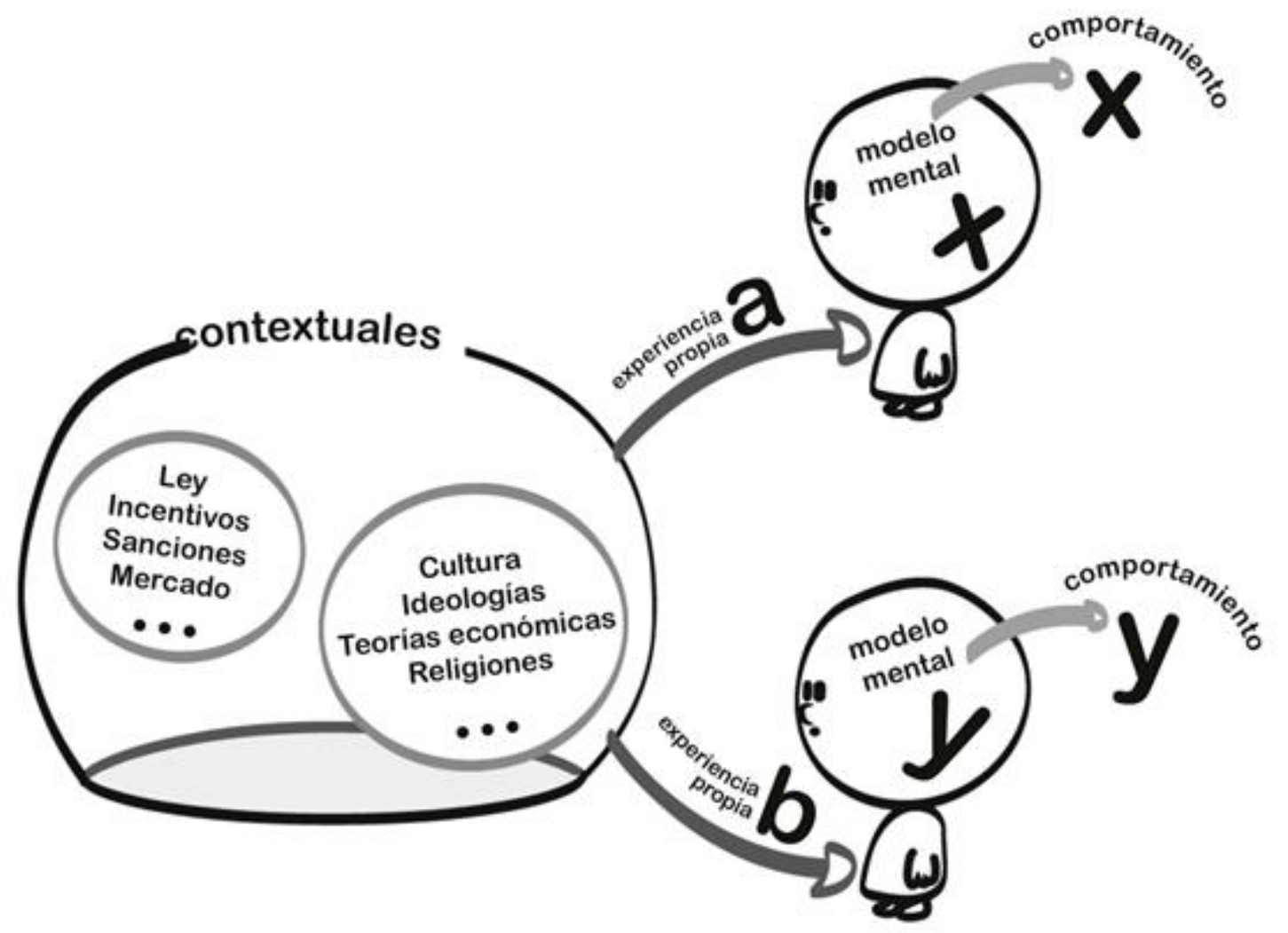

Fuente: García-Quero, 2013.

Como se aprecia en la figura 3, todas las instituciones contextuales afectan a los individuos mediante la experiencia particular de cada uno en la interacción con ellas. Así determinadas cuestiones que pueden tener un impacto irrelevante para unas personas, pueden ser hechos nota-

\footnotetext{
${ }^{13}$ El concepto original fue introducido por Sperry (1991) y hace referencias a la "causación descendente". Para ver una discusión más exhaustiva sobre el mismo ver Hodgson (2003, 2004a, 2004b).

${ }^{14}$ Hodgson (2007), apoyándose en Veblen, afirma que los hábitos ("enabling" en nuestra propuesta) se forman cuando a través de mecanismos (restricciones, incentivos, convenciones, imitaciones) los individuos repiten comportamiento y pensamientos hasta interiorizarlos. Para una explicación del mecanismo de formación de los hábitos puede verse en Hodgson y Knudsen (2004).
} 
bles en la vida de otras. De la misma manera, las influencias del contexto en los comportamientos individuales varían en función de la posición, el poder y el interés del individuo. A pesar de las diferencias en el modo en que los individuos asimilan las instituciones, determinadas influencias son experimentadas de forma similar por personas que comparten una trayectoria común. Así, creando un tipo concreto de instituciones "contextuales", fomentando una ideología particular o castigándose y premiándose un tipo u otro de actuaciones, puede lograrse que las preferencias y motivaciones de los individuos converjan y lleven a los mismos comportamientos.

La cuestión clave reside en comprender qué ocurre cuando se interrelacionan contextos, instituciones, motivaciones y comportamientos. No hay duda de que cambiando el sistema de incentivos se modifican los comportamientos, pero lo relevante, en todo caso, es investigar qué lleva a un individuo a interiorizar ese incentivo, hasta el punto de modificar su "enabling" y sus modelos mentales. La investigación debe extenderse a la indagación de las características y la forma que deben adoptar las instituciones para lograrlo, su permanencia en el tiempo y su intensidad, o las características del contexto para conseguir que confluyan las motivaciones de un grupo de personas. Conocer cómo opera este entramado y las relaciones existentes dentro de él, permitiría guiar los comportamientos individuales hacia actividades con un alto grado de retorno social, capaces de incrementar el nivel de bienestar colectivo.

La EPI cuestiona la idea de la existencia de buenas instituciones subrayando que la efectividad de las instituciones y del cambio institucional depende del equilibrio entre instituciones contextuales e individualizantes. Esto significa que las instituciones no se pueden cambiar ni trasplantar mediante acciones políticas que nieguen la diversidad institucional e ignoren el marco histórico, político y social de los países. Puesto que las instituciones contextuales difieren según el marco en el que operan, y las "individualizantes" según el individuo, los resultados de una institución con igual forma pueden ser muy diferentes.

Las instituciones gobiernan la manera en que los intereses políticos se organizan, así como las reglas de funcionamiento (jerarquía de poder, reglas electorales, comportamiento cargos públicos, tipo de votaciones, etc.). Al partir de unas instituciones "constitutivas", se asume que éstas condicionan la política afectando a las motivaciones individuales y, por ende, a los comportamientos de los gobernantes (Chang y Evans, 2000). Según esto, la corrupción de la clase política dependería de la conjunción del sistema de incentivos que produce el entorno (instituciones "contextuales") y de las motivaciones y preferencias de los propios políticos (instituciones "individualizantes").

\section{Reflexiones finales}

El fracaso de las teorías convencionales para abordar la problemática del desarrollo y el creciente interés por las instituciones está favoreciendo la recuperación de una literatura económica tradicionalmente ignorada. La Economía Política Institucional (EPI) es la dimensión institucional de un proyecto en curso cuya pretensión es refutar la actual ortodoxia desde el análisis histórico, y, en particular, desde la historia de la política económica y social. La EPI sostiene que las instituciones forman parte de las motivaciones, los individuos las interiorizan y se cambian a sí mismos, interactúan con el mundo mediante un esquema que está en continua transformación. Esta interpretación de las instituciones nos permite clasificarlas en dos tipos: "instituciones individualizantes" (forman parte de los modelos mentales de las personas) e "instituciones contextuales". Ambas se relacionan bidireccionalmente afectando a las motivaciones y los comportamientos de los individuos mediante un proceso endógeno.

Partir de esta visión de las instituciones exige un giro conceptual en la interpretación del mercado, el Estado y el cambio institucional. El mercado es una de tantas instituciones "contextuales" que conforman el capitalismo, porque a través de él se organiza y distribuye la actividad económica (recursos productivos, trabajo y capital). Los mercados perfectos no existen, por lo que la función de los Estados tiene que encaminarse a determinar los objetivos a conseguir en cada mercado y crear las instituciones necesarias para alcanzarlos. En este sentido, el éxito o el fracaso de la intervención estatal y del cambio institucional dependerán del marco producido por la interacción entre instituciones contextuales e individualizantes. 
El debate sobre estas problemáticas debe extenderse hacia la interrelación de contextos, instituciones, motivaciones y comportamientos. La investigación debe avanzar en las características, la forma o la intensidad que deben adoptar las instituciones para lograr su permanencia en el tiempo. El estudio de los procesos económicos exige restaurar una cosmovisión holística y dinámica frente a la estática actual, ampliar sus fronteras e impulsar una apertura metodológica. Economía y sociedad son sistemas dinámicos y complejos que cambian y se sustentan en interrelaciones sociales de dominación, conflicto, interés y cooperación. Los recursos naturales, la tecnología, la cultura, las instituciones, el poder, la propiedad, la ética o la acción colectiva definen contextos en los que individuos y grupos sociales toman decisiones que afectan al entorno y a los propios individuos. La comprensión de cómo operan estas estructuras y las relaciones existentes entre ellas, ayudaría a guiar los comportamientos colectivos hacia actividades con un alto grado de retorno social y con positivos efectos sobre el bienestar de las personas.

\section{Bibliografía}

Albert, Michel. 1991. Capitalism vs. Capitalism. New York, Four Walls Eight Windows.

Alt, Jamen. y Shepsle, Kenneth. (eds.) 1990. Perspectives on Positive Political Economy. Cambridge, Cambridge University Press.

Bardhan, Pranab. 2001. Deliberative Conflicts, Collective Action and Institutional Economics. En Meier, G. and Stiglitz J. (eds.), Frontiers of Development Economics: the Future in Perspective. New York, Oxford University Press, 269-290.

Berger, Suzanne. y Dore, Ronald P. 1996. National Diversity and Global Capitalism. New York, Cornell University Press.

Block, Fred. 2000. Deconstructing capitalism as a system. Rethinking Marxism, 12 (3), 83-98.

Bronfenbrenner, U. 1986. Ecology of the family as a context for human development: Research perspectives. Developmental Psychology, 22 (6), 723-742.

Bronfenbrenner, Urie and Morris, Pamela. 1998. The ecology of developmental processes. En Damon, W. y Lerner, R.M. (eds.), Handbook of child psychology, vol. 1, 993-1023. New York, John Wiley and Sons, Inc.

Buchanan, James. 1986. Liberty, Market and State. Brighton, Wheatsheaf Books Ltd.

Burlamaqui, Leonardo; Castro, Ana Celia. y Chang Ha-Joon. (ed) (2000). Institutions and the role of State. Chentelham, Edward Elgar.

Burton, John. 1983. Picking Losers...?: The Political Economy of Industrial Policy. London, Institute of Economic Affairs.

Case, Robbie. y Okamoto, Yukari. 1996. The role of central conceptual structures in the development of children's thought. Monographs of the Society for Research in Child Development, 246 (6), 1-26

Chang, Ha-Joon. 2011. Institutions and Economic Development: Theory, Policy and History. Journal of Institutional Economics, 7(4), 473-498.

Chang, Ha-Joon. (ed.). 2007. Institutional Change and Economic Development. London, Anthems Press.

- Chang, Ha-Joon. 1994a. State, Institutions, and Structural Change, Structural Change and Economic Dynamics, 5(2), 293-313.

- 1994b. The Political Economy of Industrial Policy. London and Basingstoke, Macmillan.

- 2000. An Institutionalist Perspective on the Role of the State -Towards an Institutionalist Political Economy. En Burlamaqui, L.; Castro, A. y Chang, H-J. (eds.), Institutions and the Role of the State, 3-26. Aldershot, Edward Elgar.

- 2002a. Breaking the Mould - An Institutionalist Political Economy Alternative to the NeoLiberal Theory of the Market and the State. Cambridge Journal of Economics, 26 (5), 539559.

- 2002b. Kicking away the ladder: development strategy in historical perspective. London, Anthem Press.

- 2005. Globalization, Global Standards and the Future of East Asia. Global Economic Review, 34(4), 363-378. 
Chang, Ha-Joon y Evans, Peter. 2005. The Role of Institutions in Economic Change. En Dymski Gary y Da Paula, Silvina (eds.), Reimagining Growth. London, Zed Press, 99-129.

Chang, Ha-Joon. y Rowthorn, Rowthorn 1995. Role of the State in Economic Change - Entrepreneurshipand Conflict Management. En Chang, H.-J. y Rowthorn, R. (eds.), The Role of the State in Economic Change. Oxford, Oxford University Press, 31-50.

Di John, John. 2004. The Political Economy of Industrial Policy in Venezuela, 1920-1998. PhD dissertation, Faculty of Economics University of Cambridge.

Ellerman, David. 1999. Helping Others to Help Themselves: The Challenge of AutonomyCompatible Development Assistance.Washington DC, World Bank.

Epstein, Gerald. 2006. Central Banks as Agents of Economic Development. WIDER Research Papar, 2006-54. Helsinki, UNU-WIDER.

Evans, Peter. 1995. Embedded Autonomy: States and Industrial Transformation. Princeton, Princeton University Press.

- 2007. Extending the institutional turn: property, politics, and development trajectories. En Chang, Ha-Joon (ed.), Institutional Change and Economic Development. London, Anthems Press, 35-52.

Flavell, John H. 1992. Cognitive Development: Past, Present and Future. Developmental Psychology, 28 (2), 191-204.

Frey, Bruno. 1997. Not Just for the Money: An Economic Theory of Personal Motivation. Cheltenham, Edward Elgar.

Galbraith, James K. 1955. The affluent society. Boston, Houghton Mifflin.

- (1967). The new industrial State. Boston, Houghton Mifflin.

- (1987). A history of economics: The past as the present. London, H. Hamilton.

García-Quero, Fernando. 2013. Desarrollo y economía política institucionalista: una reinterpretación del comportamiento, el estado, la política y el cambio institucional. Tesis doctoral. Granada, Editorial Universidad de Granada.

García-Quero, Fernando y López Castellano, Fernando. (2011). Other institutionalism for Development Studies, Real-world Economics Review, 58(12), 126-133.

Greif, Avner. 2006. Institutions and the path to modern economy: lessons from medieval trade. Cambridge, Cambridge University Press.

Hayek, Friedrich. 1949. Individualism and Economic Order. London, Routledge \& Kegan Paul.

Hodgson, Geoffrey. 1988. Economics and Institutions: a manifesto for a modern institutional economics. Cambridge, Policy Press.

- 1993. Economics and Evolution. Cambridge, Policy Press.

- (1998). The Approach of Institutional Economics, Journal of Economic Literature, 36 (1), $166-92$.

- 2001. How Economics Forgot History: The Problem of Historical Specificity in Social Science. London, Routledge.

- 2004a. The Evolution of Institutional Economics: Agency, Structure and Darwinism in American Institutionalism. London, Routledge.

- 2004b. Darwinism, causality and the social sciences, Journal of Economic Methodology, 2 (2), 175-194.

- 2006. What are Institutions? Journal of Economic Issues, 40 (1), 1-25.

- 2007. The Revival of Veblenian Institutional Economics, Journal of Economic Issues; 41 (2), 325-340.

Hodgson, Geoffrey. y Knudsen, Thorbjørn. 2004. The Complex Evolution of a simple Traffic convention: the functions and implications of habit, Journal of Economics Behaviour and Organizations, 54 (1), 19-47

Kapp, K.William. 1968. In Defense of Institutionalism. Swedish Journal of Economics, 70 (1), $1-18$.

Lazonick, William. 2006. Corporate Governance, Innovate Enterprise, and Economic Development, WIDER Research Paper 2006-71, Helsinki, UNU-WIDER.

- 1991. Business Organization and the Myth of the Market Economy. Cambridge, Nueva York, University Press. 
Lewis, Orion y Steinmo, Sven. 2011. Tomemos en serio la evolución: Análisis institucional y teoría evolutiva. Revista de Economía Institucional, 13 (24), 111-151.

List, Friedrich. 1885 [1841]. The National System of Political Economy. London, Green and Company.

López Castellano, Fernando y García-Quero, Fernando 2012. Institutional approaches to economic development: The current status of the debate, Journal of Economic Issues, 46 (4), 921-940.

- 2013. Instituciones y desarrollo: perspectivas desde la heterodoxia. En Arias, X. C. y Caballero, G. (eds.), Nuevo institucionalismo: gobernanza, economía y politicas públicas, 81-102. Madrid, CIS-Centro de Investigaciones Sociológicas.

Mayhew, Anne. 1989. Contrasting origins of the two institutionalisms: the social science context. Review of Political Economy, 1(3), 319-333.

March, James. y Olsen, Johan. 1989. Rediscovering Institutions: The Organizational Basis of Politics. New York, The Free Press.

Mantzavinos, Chrysostomoa; North, Douglass. y Shariq, Syed. 2004. Learning, institutions and economic performance. Perspectives on Politics, 2 (1), 75-84

North, Douglass. 1990. Institutions, Institutional change and Economic Performance. New York, Cambridge University Press.

- 2005. Understanding the Process of Economic Change. Princeton, Princeton University Press.

Olson, Mancur. 1996. Big bills left on the sidewalk: why some nations are rich and others poor. Journal of Economic Perspectives, 10 (2), 3-24.

Parke, Ross; Ornstein, Peter.; Riesesm, John. y Zahn-Waxler, Carolyn. 1994 A Century on Development Psychology. Washington DC, American Psychological Association.

Piaget, Jean. 1977. The development of thought: equilibration of cognitive structures. California, Viking Press University of California.

Polanyi, Karl . 1944. The Great Transformation. Boston, Massachusetts, Beacon Press.

Reinert, Erik. 1995. Competitiveness and its predecessors: a 500 years Cross national Perspective. Structural Change and Economic Dynamics, 6(1), 23-42.

- 2004. Globalization, Economic Development and Inequality: An alternative Perspective. Cheltenham, Edward Elgar.

- 2006. Institutionalism Ancient, Old and New: A Historical Perspective on Institutions and Uneven Development. World Institute for Economic Development Research (UNU-WIDER), United Nation University, Research Paper 2006/77.

— 2007. How Rich Countries Got Rich ... and Why Poor Countries Stay Poor. London, Constable \& Robinson.

Rutherford, Malcolm. 1994. Institutions in Economics-the old and the new institutionalism. Cambridge, Cambridge University Press.

Schmoller, Gustav. 1910 [1884]. The journal Schmoller's Jahrbuch. The Mercantile System and its Historical Significance. Nueva York, Mamillan.

Simon, Herbert. 1983. Reasons in Human Behaviour. Oxford, Basil Blackwell.

Sperry, Roger W. 1991. In Defense of Mentalism and Emergent Interaction. Journal of Mind and Behavior, 12 (2), 221-46.

Toboso, Fernando. 1997. ¿En qué se diferencian los Enfoques de Análisis de la vieja y la nueva Economía Institucional? Hacienda Pública Española, 143, 175-192.

Veblen, Thorstein. 1899. The Theory of the Leisure Class. An Economic Study in the evolution of Institutions. New York, Mcmillan Company.

- 1914. The instinct of Workmanship, and the State of the industrial Art. New York, Mcmicllan

Vira, Bhaskar. 1997. The political Coase theorem: Identifying differences between neoclassical and critical institutionalism, Journal of Economic Issues, 31 (3), 761-780.

Williamson, Oliver E. 2000. The new institutional economics: taking stock, looking ahead, Journal of Economic Literature, 38 (3), 595-613. 
Woo-Cumings, Meredith J. 1999. The Developmental State. New York, Cornell University Press. 